\title{
Transnationale Lohnkoordination in einem System fester Wechselkurse
}

\author{
MARTIN HÖPNER
}

Die Redaktion der WSI-Mitteilungen hat für den Debattenteil dieser Ausgabe ein ebenso wichtiges wie brisantes Thema gewählt. Denn der Euro hat die Notwendigkeit transnationaler Lohnkoordination erhöht. Und das Zustandekommen effektiver transnationaler Lohnkoordination dürfte über die Zukunft des Euro-Projekts entscheiden.

\section{Die Inflationsraten müssen synchronisiert werden}

Eine Zielsetzung der europäischen Gewerkschaften war die transnationale Lohnkoordination schon lange vor dem Euro, ohne jedoch hiermit jemals nennenswert vorangekommen zu sein (siehe die Einzelheiten in den Beiträgen von Hof mann und Schulten in diesem Heft). Was hat sich durch den Eintritt in den Euro verändert? Eine Währungsunion ist die glaubwürdigste Form eines Regimes fester Wechselkurse. Das bedeutet, dass transnationale Inflationsunterschiede nicht mehr durch nominale Auf- und Abwertungen der nationalen Währungen ausgeglichen werden können. Der Dreh- und Angelpunkt einer Währungsunion besteht daher in der Fähigkeit ihrer Teilnehmer, die nationalen Inflationsentwicklungen zu synchronisieren. Die wichtigste Determinante von Inflationsraten sind die Entwicklungen der nominalen - nicht: der realen! - Lohnstückkosten. Die nationalen Tarifpartner müssen ihre Lohnpolitiken also dahin gehend aufeinander abstimmen, dass sie mittelfristig ähnliche Inflationsraten hervorbringen.

Man stelle sich Gewerkschaftsvorsitzende aus 18 Euro-Teilnehmerstaaten vor, die jährlich in Brüssel zusammenkommen müssen, um dort auszuhandeln, welche Lohnsteigerungen sie in den anstehenden Tarifrunden gegenüber den jeweiligen Arbeitgeberverbänden ihrer Herkunftsländer durchsetzen müssen - zweifellos ein Gruselszenario. Aber etwas handhabbarer ist die Sache immerhin. Denn orientieren sich alle an derselben Lohnregel, dann sollte die Synchronisation der nationalen Preisauftriebe mittelfristig gelingen, ohne dass sich hierfür wer in die Bahn oder in den Flieger nach Brüssel setzen muss.

Und im Prinzip existiert auch die hierfür notwendige Lohnregel - nämlich in Gestalt der Zielinflationsrate der Europäischen Zentralbank, die auf weniger als, aber nahe an $2 \%$ festgelegt wurde. Wenn wir nun annehmen, dass sich jede Einheit nominaler Steigerungen der Lohnstückkosten eins zu eins in eine ebenso hohe Inflation übersetzt was empirisch in der Tat ungefähr zutrifft -, dann bedeutet dies, dass die nationalen Lohnstückkosten in jedem Land um jährlich ungefähr $2 \%$ steigen müssen (mit anderen Worten: die Zielmarken für die Nominallohnsteigerungen ergeben sich aus Produktivitätszuwächsen plus Zielinflation). Zudem wird das Ganze dadurch erleichtert, dass die Marke nicht Jahr für Jahr zielgenau eingehalten werden muss. Fehlentwicklungen lassen sich in den Folgejahren korrigieren. Mittelfristig aber ist der lohnpolitische Beitrag zur Synchronisation der Inflationsraten essenziell. Das ist der Preis der Währungsunion.

Die Crux des Ganzen ist die Orientierung an der Inflationsrate der Europäischen Zentralbank (EZB) statt der nationalen Inflationsrate, die die nationalen Gewerkschaften typischerweise neben der Produktivitätsentwicklung in Rechnung stellen. Denn eine Orientierung an der nationalen Kennziffer würde genau das bewirken, was in einer Währungsunion nicht geschehen darf: Die Inflationsdifferenzen würden verstetigt und die preisliche Wettbewerbskraft der teilnehmenden Länder würde von Jahr zu Jahr weiter auseinanderklaffen, ohne dass das Instrument der nominalen Wechselkursanpassung zur Korrektur der so entstandenen Disparitäten zur Verfügung stünde. Irrelevant wird die europäische Zielinflation als Orientierungsmarke nur, wenn die Synchronisation der Inflationsraten bereits erfolgt ist - bestenfalls auf dem Niveau der Zielinflation, bei dem für die EZB keine Veranlassung besteht, die Konjunkturen durch Zinserhöhungen zu bremsen. Aber diese Synchronisation gilt es ja gerade herzustellen.

\section{Die verlorene Wette}

Eine Währungsunion ist gewissermaßen eine gewagte Wette. Der Eintritt in den Euro vertieft die wirtschaftliche Integration, stabilisiert die Erwartungen der Wirtschaftsteilnehmer und verleiht dem Integrationsraum mit der gemeinsamen Währung ein gewichtiges Symbol. Gleichzeitig wetten die Mitglieder der Währungsunion darauf, das Instrument der nominalen Wechselkursanpassung künftig nicht mehr zu brauchen. Diese Wette wurde verloren. Um ungefähr kumuliert $22 \%$ hätten die nominalen Lohnstückkosten der Euro-Teilnehmerstaaten während der ersten zehn Eurojahre (1999-2008, danach brach die EuroKrise aus) steigen müssen, wäre die aus der Zielinflationsrate folgende Lohnregel von allen eingehalten worden. Tatsächlich aber sanken die nominalen Lohnstückkosten in Deutschland um $2 \%$, in Österreich stiegen sie um 6\%, in Frankreich um $20 \%$, in Italien um $28 \%$ und in Spanien um $36 \%$.

Das hatte, wie wir wissen, dramatische Folgen. Die preislichen Wettbewerbskräfte der teilnehmenden Länder verschoben sich quer über den europäischen Kontinent, genauer: von Süd nach Nord. Als der Schock der amerikanischen Immobilienkrise einsetzte, begannen die Kapitalmarktteilnehmer an der Fähigkeit der Länder mit Leistungsbilanzdefiziten zu zweifeln, ihre Defizite dauerhaft zu finanzieren. Wichtig ist an dieser Stelle die Verklammerung von Wettbewerbs- und Refinanzierungskrise. Eine zu laxe Haushaltspolitik hat es beispielsweise in Spanien nicht gegeben, trotzdem gerieten auch die spanischen $\mathrm{Fi}$ nanzen in den Sog der Wettbewerbskrise.

Wie konnten sich die nationalen Lohnstückkosten derart auseinanderentwickeln? Erstens, die Lohnstückkosten folgten den nationalen Wachstumspfaden, die ihrerseits durch den Euro auseinandergetrieben wurden. In den Südlän dern setzten kreditgetriebene Wachstumsschübe ein, während sich der Norden mit für seine Ökonomien zu hohen Realzinsen konfrontiert 
sah. Zweitens, die Euro-Teilnehmerländer verfügen über höchst unterschiedliche Regime der Lohnaushandlung. In den Ländern mit vergleichsweise koordinierten Tarifsystemen waren die Lohnauftriebe geringer, als man es aufgrund der dortigen Wachstumsraten erwarten würde, und in den Ländern mit vergleichsweise unkoordinierten und dezentralisierten Tarifsystemen höher, als es die dortigen Wachstumsraten nahegelegt hätten. Entscheidend ist an dieser Stelle: Es existierte keine transnationale Lohnkoordination, die in der Lage gewesen wäre, diesen auseinandertreibenden Schubkräften der nominalen Lohnstückkosten Einhalt zu gebieten.

Zudem darf eine dritte Ursache für die Auseinanderentwicklung der Lohnstückkosten nicht vernachlässigt werden. Sie betrifft den deutschen Sonderweg. In Deutschland war der Druck auf die Lohnstückkosten noch größer, als man es auf Grundlage der Kombination aus vergleichsweise niedrigen Wachstumsraten - bis zum Ausbruch der Eurokrise war Deutschland alles andere als ein Gewinner des Euro! - und vergleichsweise koordinierten Arbeitsbeziehungen erwartet hätte. Die Ursache hierfür liegt in der arbeitsmarktund sozialpolitisch begünstigten Verschärfung der Lohnkonkurrenz zwischen Beschäftigten und Arbeitslosen und der Spaltung der Belegschaften in Kerne und Ränder, zudem in der sinkenden Tarifbindung in wachsenden Bereichen, wie beispielsweise den niedrig produktiven Dienstleistungen. Skeptisch bin ich gegenüber Behauptungen, intendierte Lohnzurückhaltung habe es in Deutschland bis zum Ausbruch der Eurokrise und danach nicht gegeben. Die gesamtwirtschaftlichen Effekte dieser vorsichtigen Lohnpolitik wurden aber radikalisiert durch Faktoren, die die Gewerkschaften weder im Griff noch gewollt hatten. Auch hier gilt: Eine transnationale Lohnkoordination, die diesen Entwicklungen hätte Einhalt gebieten können, existierte nicht.

Nicht glücklich bin ich in diesem Zusammenhang mit den Einwänden Hofmanns und Schultens, die durch - intendierte oder nicht intendierte - Lohnzurückhaltung erzielten Kostenvorteile Deutschlands seien für die Eurokrise wenig erheblich, weil die Preiselastizitäten auf den internationalen Märkten gering seien und die Südländer ohnehin über mangelhafte Wirtschaftsstrukturen verfügen würden. Es sei noch einmal betont: Die vorsichtige Lohnpolitik der Gewerkschaften war nur für einen Teil der gesunkenen deutschen Lohnstückkosten verantwortlich, die Reformen der Schröder-Ära besorgten ein Übriges. Für das Ergebnis aber ist dieser Umstand unerheblich. Deutschland hat sich aus seiner Rolle als erstes
Opfer des Euro befreit, indem es viele seiner Nachbarn niederkonkurrierte - bis an den Punkt, an dem die Fähigkeit dieser Länder prekär wurde, ihre Importüberschüsse weiter zu finanzieren. Diesen Ländern nun hinterherzurufen, dass Kosten und Preise keine Rolle spielen - das wäre nicht gut. Auch der Verweis auf unterentwickelte Wirtschaftsstrukturen in Ländern wie Griechenland überzeugt mich nicht, denn was der Ökonom in solchen Konstellationen doch eigentlich erwarten würde, sind wirtschaftliche Aufholeffekte - so wie wir sie derzeit in der Türkei erleben, deren Wirtschaftsstruktur gewiss nicht „moderner“ ist als jene Griechenlands, die aber anders als Griechenland keinem falschen Währungsregime unterworfen ist.

Nun ist das Kind in den Brunnen gefallen und die Anforderungen an transnationale Lohnkoordination haben sich damit zusätzlich potenziert. Denn die Orientierung an der Zielinflationsrate der EZB reicht nun nicht mehr. Eine europäische Lohnkoordination müsste nunmehr auf die Wiederangleichung der erfolgten realen Wechselkursverzerrungen hinwirken. In welchem Zeitraum kann und soll dies erfolgen? Und wer übernimmt welchen Teil der Anpassungslast? Allein der Süden, der so lange durch die Folterkeller von Sozialabbau, Arbeitsmarktderegulierung und "union busting" gezerrt wird, bis sinkende nominale Lohnstückkosten das Preisniveau der dortigen Länder auf das Ausgangsniveau gesenkt haben? Oder auch der Norden, der den Süden seinerseits durch Lohninflationierung von einem Teil der Anpassungslast befreien könnte? Um dies auszuhandeln, ist Lohnkoordination im engeren Sinne erforderlich. Übernähme in dieser Überganszeit zudem die EZB die Aufgabe, die Risikoaufschläge auf Staatsanleihen weiter auf niedrigem Niveau anzugleichen, dann wäre ein Ausgang aus der Eurokrise gefunden. ${ }^{1}$

\section{Die Unwahrscheinlichkeit effektiver Koordination}

Genau an diesem Punkt ist Pessimismus angezeigt. Die EZB ist nicht das Problem. Aber die Lohnkoordination. Transnational lässt sich effektiv nur das koordinieren, was auch national koordiniert ist. Das ist eine Voraussetzung, die im Euro nicht nur ex ante nicht erfüllt war - die Troika-Politik hat die Tarifsysteme der Südländer zudem so weit demoliert, dass wir von dieser Voraussetzung weiter entfernt sind als jemals zuvor. Aber selbst wenn wir uns dieses Problem einmal im Gedankenexperiment wegdenken: Wie ausgeprägt ist die Bereitschaft der Exportgewerkschaften des Nordens, ihre Lohnpolitik künftig an einer europäischen Zielinflation statt an der nationalen Inflationsrate $\mathrm{zu}$ orientieren? Hofmann problematisiert diesen Umstand zu Recht, während Schulten, wenn ich ihn richtig verstehe, für die Beibehaltung der nationalen Inflationsrate als Orientierungsmarke plädiert - was ja aus einer nationalen Perspektive auch Sinn macht, aber in einer Währungsunion nicht funktional sein kann, weil es die transnationalen Inflationsdivergenzen perpetuiert. Vor allem aber müssen wir uns fragen: Wären die deutschen, finnischen, österreichischen und belgischen Gewerkschaften bereit, gezielt zu inflationieren, um den Süden von Deflationierungsdruck zu entlasten?

Namentlich, wären die deutschen Gewerkschaften hierzu bereit oder angesichts starker sozialpartnerschaftlicher Wettbewerbskoalitionen in den Großunternehmen und abnehmender Tarifbindung bei kleinen und mittleren Unternehmen auch nur potenziell in der Lage? Mit einer solchen Strategie würden sich die Exportgüter Deutschlands nicht nur gegenüber den Preisniveaus der anderen Euro-Teilnehmer verteuern - eben dies wäre ja der Sinn der Übung -, sondern auch gegenüber Wettbewerbern außerhalb des Euros. Sollte ich eine Zahl nennen, würde ich antworten: Diese Bereitschaft und Fähigkeit geht gegen Null. Eine Strategie der gezielten Verteuerung der deutschen Exportgüter könnte und würde, selbst wenn sich die Exportgewerkschaften auf diese verständigten, die Wettbewerbskoalitionen in den Exportunternehmen nicht mittragen. Neue Schübe an Lohnzurückhaltung würden einsetzen, sobald die Exportsektoren unter Druck geraten.

Zeugt diese Sicht der Dinge von überbordendem Pessimismus? Vielleicht vermag ich mir einfach nicht vorzustellen, dass das, was in der Vergangenheit stets scheiterte und für das es derzeit keine Anzeichen gibt, in Zukunft funktionieren könnte. Bis auf Weiteres aber lautet meine Einschätzung: Die IG Metall wird ihre Lohnpolitik nicht an der Zielinflation der EZB - oder einer anderen europäischen Zielinflation, wenn man sich denn transnational auf diese verständigen würde -, orientieren. Und sie würde eher den Austritt aus dem Euro forcieren, als gezielt lohnpolitisch zu inflationieren, um den derzeit überdimensionierten deutschen Exportsektor abzu-

1 Der in den europäischen Volkswirtschaften wirksame Effektivzins wäre mit diesen Interventionen freilich noch nicht angeglichen. Dieses Problem ist in den Euro strukturell eingepflanzt. 
schmelzen. Der Euro lädt die Lohnpolitiken mit Anpassungslasten auf, die die Steuerbarkeit makroökonomischer Lohnaggregate systematisch überfordern. Damit aber ist dem festen Wechselkursregime die Grundlage entzogen. Seien wir ehrlich: Der Euro war von Anfang an eine schlechte Idee.

\section{Bitte keine Ermächtigung der Kommission}

Ich schließe mit zwei Anmerkungen zur europäischen Politik. Erstens, Hofmann plädiert (idH, S. 312) dafür, den neuen makroökonomischen Verfahren „ein ebenso verbindliches europäisches Semester der Gewerkschaften, also ein System sozialer Kennziffern und konkreter Zielsetzungen der Lohnpolitik gegenüber[zu]stellen“. Ist dies so zu verstehen, dass man seinen Frieden mit den europäischen Verfahren geschlossen hat und diese lediglich noch um gewerkschaftliche Anbauten ergänzen will? Ist die Tatsache akzeptiert, dass die europäischen Organe „falsche“ Lohnpolitik künftig sanktionieren werden? Sowohl Hofmann als auch Schulten stellen klar, dass sich die europäischen Gewerkschaften neben europaweiten Lohnregeln sogenannte Umverteilungskomponenten vorbehalten werden. Nun nehmen wir einmal an, dass diese Umverteilungskomponenten in transnational koordinierter Weise eingefordert, also keine für ein festes Wechselkursregime bedenklichen Inflationsdifferenzen hervorbringen würden. ${ }^{2}$ Gleichwohl würden diese Komponenten im Rahmen des Verfahrens zur Erkennung und Vermeidung makroökonomischer Ungleichgewichte sanktioniert! Hier stellt sich die Frage, wer denn die Trägerinnen und Träger der zweifellos notwendigen transnationalen Lohnkoordination sein könnten und sollten - die Gewerkschaften selbst oder eine Behörde, die „falsche“ Lohnpolitik mit Strafen belegt?

Diese - zugegebenermaßen rhetorisch gemeinte - Frage führt mich zu meiner zweiten Anmerkung. Denn bei Licht besehen beantworten Hofmann und Schulten die Frage recht unterschiedlich. Die Trägerinnen und Träger der Lohnkoordination, so schreibt Hofmann, „können allein die Gewerkschaften sein“ (ebd., S. 311). Schulten hingegen beklagt die Existenz der Sperrklausel in Art. 153 Abs. 5 AEUV, die alle Fragen des Arbeitsentgelts und des Arbeitskampfrechts von den sozialpolitischen Kompetenzen der EU ausnimmt. Ich vermute an dieser Stelle, dass seine Überlegungen auf eine europäische Rahmenrichtlinie zielen, die die gewerkschaftliche Lohnkoor- dination mit größerer Verbindlichkeit ausstatten könnte. So oder so kann ich nur nachdrücklich davor warnen, die Kommission mit einer Befug nis zur Vorlage von Richtlinien- und Verordnungsvorschlägen in Lohnfragen ausstatten zu wollen. Die Stellungnahmen der Kommission zu Viking und Laval, ihr Umgang mit den Lohnsystemen der Krisenländer und die vorgeschlagene Monti-II-Verordnung zur Errichtung eines Erfassungsregimes für Arbeitskämpfe zeigen un missverständlich, was die Kommission mit einer solchen Befugnis anstellen würde. Nur Dank der Sperrklausel in Art. 153 AEUV erntete die Kommission auf Monti II Subsidiaritätsklagen, die sie zur Rücknahme ihrer Vorschläge bewegten. Schulten und Müller haben die Vorstellungen der Kommission zur Umgestaltung der mitgliedstaatlichen Tarifsysteme erst jüngst hervorragend dokumentiert. ${ }^{3}$ Sie verdienen nur eine Bezeichnung: Sie sind Kampfansagen an oberhalb der Unternehmensebene angesiedelte Lohnfindung. Der Kommission in dieser Situation ein zusätzliches Instrument anzubieten, mit dem sie ihre Ziele im Prinzip erreichen könnte - das wäre ein schwerer strategischer Fehler.

Ich argumentiere an dieser Stelle mit besonderem Nachdruck, weil ich die Gefahr eines strategischen Eigentors auch in einer anderen Forderung angelegt sehe: in dem auch in Schultens Stellungnahme auftauchenden Ruf nach „europaweiten Normen für ein angemessenes Mindestlohnniveau" (idH, S. 316). Genau diese Normsetzungskompetenz hat die EU derzeit nicht (und am Rande sei erwähnt, dass mir unverständlich ist, wie die europäischen Sozialdemokraten unbeschadet dessen mit der Forderung nach einem europäischen Mindestlohn Wahlkampf betreiben können - so organisiert man Europafrust). Auch dieser Ruf läuft daher entweder auf eine versuchte Umgehung, oder aber eine gewünschte $\mathrm{Ab}$ schaffung der Sperrklausel des Art. 153 Abs. 5 AEUV hinaus. Vor beidem kann ich nur warnen. Der Kampf um Mindestlöhne ließe sich europäisch koordinieren (wenn diese Lohnuntergrenzen

2 Ich vernachlässige an dieser Stelle, dass in einer solchen Konstellation mit zinspolitischen Reaktionen der Europäischen Zentralbank zu rechnen wäre.

3 Schulten, Th./Müller, T. (2013): Ein neuer europäischer Interventionismus? Die Auswirkungen des neuen Systems der europäischen Economic Governance auf Löhne und Tarifpolitik, in: Wirtschaft und Gesellschaft 39 (3), S. 291-321. auch schwerlich die Eurokrise auflösen dürften) Aber die Forderung nach einer Richtlinie stellt die europäische Kompetenzordnung auf den Kopf. Wer eine Mindestlohnrichtlinie fordert, wird Monti III ernten.

\section{AUTOR}

MARTIN HÖPNER, apl. Prof. Dr., ist Wissenschaftler am Max-Planck-Institut für Gesellschaftsforschung (MPIfG), Köln. Arbeitsschwerpunkt: Vergleichende Politische Ökonomie.

@mh@mpifg.de 